\title{
Kawasaki disease, Mycoplasma pneumoniae infection and anaplastic large cell lymphoma: An uncommon association
} \author{
Saoussen Abroug ${ }^{1}$, Abdelaziz Harbi ${ }^{1}$ \\ ${ }^{1}$ Department of Paediatrics, Sahloul Hospital, Sousse, Tunisia \\ ${ }^{2}$ Laboratory of Bacteriology-Virology, Sahloul Hospital, Sousse, Tunisia \\ ${ }^{3}$ Department of Cytopathology, Farhat Hached Hospital, Sousse, Tunisia \\ Email: jalel_chemli@yahoo.fr
}

Jalel Chemli ${ }^{1}$, Saida Hassayoun ${ }^{1}$, Soumaya Ketata ${ }^{2}$, Ajmi Houda $^{1}$, Moncef Mokni ${ }^{3}$, Noura Zouari ${ }^{1}$,

Received 20 October 2011; revised 13 February 2012; accepted 19 July 2012

\begin{abstract}
Kawasaki disease (KD) is an acute febrile systemic vasculitis occurring predominantly in young children less than 5 years of age. Although imperfectly known, the aetiopathogenesis of KD would be secondary to immunological abnormalities that could constitute a favourable terrain for neoplasms. We report on a case in a 2-year-old girl who presented clinical manifestations compatible with Kawasaki disease complicated by coronary aneurysm. Aetiopathological investigations revealed $M$. pneumoniae infection as specific IgM were present in the serum (Elisa). The patient was initially treated by intravenous immunoglobulins (IVIG) and aspirin to anti-inflammatory dose. Following a few days of desquamation, resolution of the symptomatology occurred. Four weeks later she developed disseminated tumorous syndrome. Lymph node biopsy revealed massive infiltration by large cells lymphomatous proliferation. Histologic and immunophenotypic findings were characteristic of $\mathrm{ALK}^{+}{ }^{+}$anaplastic large cell lymphoma. Four weeks later, the patient died from a severe nosocomial infection complicated by septic shock. Our observation is the first cases describing the association between anaplastic large cell lymphoma, KD and $M$. pneumoniae. Immunologic disorder due to $\mathrm{KD}$ and $M$. pneumoniae infection may play probably a central role for malignancy.
\end{abstract}

Keywords: Kawasaki Disease; Mycoplasma pneumoniae; Anaplastic Large Cell Lymphoma; Immunological Abnormality

\section{INTRODUCTION}

Kawasaki disease (KD) is a systemic vasculitis that occurs predominantly in infants and young children, first described in Japan in 1967 by Tomisaku Kawasaki [1].
The etiology and pathogenesis of KD still remain unknown. However, immunological abnormalities in the acute phase of $\mathrm{KD}$ are well documented and may be pathogenic in the known cardiac and noncardiac complications of the disease. Despite the well-known association between acquired immunodeficiency states and neoplasia, there are only a few reports of malignant neoplasms accompanying or following the onset of this disease [2-4]. We report here an exceptional case of KD associated with Mycoplasma pneumoniae infection and complicated by anaplastic large cell lymphoma in a young girl.

\section{CASE REPORT}

A 2-year-old Tunisian girl was diagnosed with Kawasaki disease after presenting with a 10-day history of fever, bilateral nonexudative conjunctivitis, erythema of the lips and oral mucosa, swelling and erythema of the palms and soles, rash, and cervical lymphadenopathy. Laboratory investigation revealed an haemoglobin concentration of $6 \mathrm{~g} / \mathrm{dL}$, white blood cell count of $15,700 / \mathrm{mm}^{3}$ (with $72 \%$ neutrophils, $24 \%$ lymphocytes and $3 \%$ monocytes) and platelet count of $750 \times 10^{3}$ cells/L. C-reactive protein was elevated to $102 \mathrm{mg} / \mathrm{L}$ and erythrocyte sedimentation rate to $65 \mathrm{~mm}$ in the first hour. Visceral laboratory investtigations (renal and hepatic function, muscular enzyme) revealed no abnormalities. Chest X-ray revealed bronchial syndrome associated with diffuse alveolar opacities. Echocardiogram showed coronary artery ectasia (left main coronary artery $5 \mathrm{~mm}$, left anterior descending artery $3.4 \mathrm{~mm}$ and right coronary artery $3.2 \mathrm{~mm}$ ). Aetiological investigations revealed $M$. pneumoniae infection as specific IgM were present in the serum (Elisa, DRG Diagnostic, Allemagne). Serologic tests for Epstein-Barr virus (EBV), rubella and cytomegalovirus (CMV) were negative. The patient was treated with intravenous immunoglobulin (IV-IG) $2 \mathrm{~g} / \mathrm{Kg}$ single infusion and started on aspirin $100 \mathrm{mg} / \mathrm{Kg} /$ day and erythromycin $(50 \mathrm{mg} /$ 
Kg/day). Following a few days of desquamation, complete resolution of the symptomatology occurred. Four weeks later, she developed tumorous syndrome with moderate hepatosplenomegaly and palpable left cervical and profound lymph adenopathies detected by the ultrasonography of the abdomen and the chest computed tomography. Examination of the bone marrow revealed only granulous hyperplasia without tumorous infiltration.

Lymph node biopsy revealed massive infiltration by large cells lymphomatous proliferation. The neoplastic elements were immunoreactive for leukocyte common antigen, CD30, ALK-1 and epithelial membrane antigen. These histologic and immunophenotypic findings were characteristic of ALK $-1^{+}$anaplastic large cell lymphoma. A combination chemotherapy with methotrexate, idarubicin, cytarabine and thiotepa was started.

Two weeks later, the patient died from a severe nosocomial infection complicated by septic shock.

\section{DISCUSSION}

Although coronary artery involvement is the feature responsible for most of the morbidity in $\mathrm{KD}$, other complications can also occur.

Our observation is particular by the association of KD with $M$. pneumoniae infection and anaplastic large cell lymphoma never described at to day. However, if linking $M$. pneumoniae to $\mathrm{KD}$ is actually considered that is a possible trigger [5-9], relationship between KD and malignancies remains unclear and controversial. The hypothesis that there may be an unrecognized peri-malignant syndrome of signs and symptoms is discussed by Murray et al. [2]. Clinicians must rely on the presence of specific clinical criteria and laboratory data, excluding other illnesses that can mimic the disease [10]. In this situation the strict diagnostic criteria of Kawasaki disease are required. However, some patients do not fulfil all major clinical criteria of the disease and are diagnosed based on echocardiogram findings. Because of the prolonged history of unexplained fever, bilateral nonexudative conjunctivitis, erythema of the lips and oral mucosa, swelling and erythema of the palms and soles, rash, and cervical lymphadenopathy, KD was considered in our patient and confirmed sonographically with coronary artery ectasia.

Inaba et al. [11] reported one case of anaplastic large cell lymphoma associated with Sjogren's syndrome suggesting that the association of vasculitis with malignancy remains possible.

The aetiology of KD is unclear. The hypothesis that Kawasaki disease is related to a bacterial superantigenic toxin has been suggested because of the reported selective expansion of $\mathrm{V} \beta 2$ and $\mathrm{V} \beta 8$ T-cell receptor families, but this theory remains controversial. Mycoplasma arthritis has been shown to produce superantigen, and it is therefore possible that other Mycoplasma organisms may do likewise. However, the relationship between KD and malignancies remains unclear. There are only a few reports of malignant neoplasms associated with KD [2-4]. This association suggests that there may be a relationship between immunological disorder in KD and the development of malignancy. In the Japanese literature eight patients (6 males and 2 females) with history of KD developed neoplasm [4]. The interval between KD and development of neoplasm is variable: 4 weeks to 14 years. Four of them died. The nature of the neoplasm is also variable: 3 cases of acute lymphocytic leukemia, 1 case of Hodgkin's disease, 1 case of schwannoma, 1 cases of giant cell tumor of the tendon sheath, 1 case of osteosarcoma and 1 case of malignant reticuloma. Seven of the eight KD patients who developed neoplasms, were treated with high-dose intravenous immunoglobulin (IVIG). It is noteworthy that lymphoid malignancies developed in five cases very shortly after the onset of KD. This suggests that lymphoid malignancies might have developed in the bone marrow and/or lymphoid tissues at the onset of KD [4]. Physiopathology of development malignancy after KD remains unclear. However, it actually admitted that immunological disorder may play probably a central role.

Striking immune perturbations occur in acute KD, including marked cytokine cascade stimulation and endothelial cell activation. Both HLA-DR ${ }^{+} \mathrm{CD}^{+}$(activated T cells) and $\mathrm{DR}^{+} \mathrm{CD} 4^{+}$cells (activated helper T cells) were significantly increased in the lamina propria of patients with acute Kawasaki disease as compared with controls. In contrast, $\mathrm{CD}^{+}$cells (suppressor/cytotoxic $\mathrm{T}$ cells) were significantly reduced in both the epithelium and the lamina propria of individuals with Kawasaki disease as compared with controls [12].

Polyclonal activation of T cells in KD as manifested by increased interleukins, tumor necrosis factor- $\alpha$ (TNF$\alpha$ ) and increased level HLA-DR ${ }^{+} \mathrm{CD}^{+}$(activated T cells) and $\mathrm{DR}^{+} \mathrm{CD}^{+}$cells (activated helper $\mathrm{T}$ cells) is well documented [13]. Moreover, many growth factors such as G-CSF (granulocyte colony-stimulating factor), M-CSF (macrophage colony-stimulating factor) [14], VEGF (Vascular endothelial growth factor) [15] in the acute phase of KD, might accelerate the development of lymphoid malignancies [16]. This immunological disorder present in the acute phase of KD may persist for a long period.

The role of infectious factor is not excluded. However, patients with conditions causing aberration of lymphocyte function such as EBV infection, human immunodeficiency virus infection (HIV) and post morrow transplantation appear to be a favourable terrain for theses neoplasms. Our patient presented M. pneumoniae infection. Extrapulmonary manifestations in $M$. pneumoniae 
infection may occur through immune dysregulation [17]. But relationship between this type of infection and malignancy remains unclear and not well documented yet. Our observation is the first case describing the association between anaplastic large cell lymphoma, $\mathrm{KD}$ and $M$. pneumoniae. Immunologic disorder due to $\mathrm{KD}$ and $M$. pneumoniae infection may play probably a central role for malignancy and especially for anaplastic large cell lymphoma.

Further, more detailed epidemiological studies may be required to clarify the relationship between malignancy, $\mathrm{KD}$ and M. pneumoniae infection.

\section{REFERENCES}

[1] Kawasaki, T. (1967) Acute febrile mucocutaneous syndrome with lymphoid involvement with specific desquamation of fingers and toes in children: Clinical observation of 50 cases. Japanese Journal of Allergology, 16, 178-222.

[2] Murray, J.C., Bomgaars, L.R., Carcamo, B. and Mahoney, D.H. Jr. (1995) Lymphoid malignancies following Kawasaki disease. American Journal of Hematology, 50, 299-300. doi:10.1002/ajh.2830500414

[3] Nakamura, Y., Yanagawa, H., Harada, K., Kato, H. and Kawasaki, T. (2002) Mortality among 139 persons with a history of Kawasaki disease in Japan. The fifth look. Archives of Pediatrics \& Adolescent Medicine, 156, 162165.

[4] Suzuki, H., Takeuchi, T., Minami, T., Shima, Y., Iwahashi, S., Shibuta, S., Uemura, S., Kounami, S., Aoyagi, N. and Yoshikawa, N. (2005) Neoplasms in three patients following Kawasaki disease. Pediatrics International, 47, 217-219.

[5] Chemli, J., Hassayoun, S., Ketata, S., Ajmi, H., Ayeche, H., Zouari, N., Abroug, S., Boujaafar, N. and Harbi, A. (2010) Kawasaki disease and Mycoplasma pneumoniae infection. Medecine et Maladies Infectieuses, 40, 717-719.

[6] Merlin, E., Al Fatuhi, H. and Crost, P. (2004) Kawasaki syndrome and Mycoplasma pneumoniae infection. Archives of Pediatrics \& Adolescent Medicine, 11, 972-979.

[7] Vitale, E.A., La Torre, F., Calcagno, G., Infricciori, G., Fede, C., Conti, G., Chimenz, R. and Falcini, F. (2010) Mycoplasma pneumoniae: A possible trigger of Kawasaki disease or a mere coincidental association? Report of the first four Italian cases. Minerva Pediatrica, 62, 605-607.
[8] Wang, J.N., Wang, S.M., Liu, C.C. and Wu, J.M. (2001) Mycoplasma pneumoniae infection associated with Kawasaki disease. Acta Paediatrica, 90, 594-595. doi:10.1111/j.1651-2227.2001.tb00810.x

[9] Lee, M.N., Cha, J.H., Ahn, H.M., Yoo, J.H., Kim, H.S., Sohn, S. and Hong, Y.M. (2011) Mycoplasma pneumoniae infection in patients with Kawasaki disease. Korean Journal of Pediatrics, 54, 123-127.

[10] Newburger, J.W., Takahashi, M., Gerber, M.A., Gewitz, M.H., Tani, L.Y., Burns, J.C., et al. (2004) Diagnosis, treatment, and long-term management of Kawasaki disease: A statement 158 for health professionals from the Committee on Rheumatic Fever, Endocarditis and Kawasaki Disease, council on cardiovascular disease in the young, American Heart Association. Circulation, 110, 2747-2771. doi:10.1161/01.CIR.0000145143.19711.78

[11] Inaba, H., Kawasaki, H., Nakamura, S., Yamamoto, H., Kaneko, Y., Satake, N., Komada, Y., Ito, M. and Sakurai, M. (1998) Anaplastic large cell lymphoma associated with Sjögren’s syndrome. Leukemia and Lymphoma, 32, 183-188.

[12] Nagata, S., Yamashiro, Y., Maeda, M., Ohtsuka, Y. and Yabuta, K. (1993) Immunohistochemical studies on small intestinal mucosa in Kawasaki disease. Pediatric Research, 33, 557-563. doi:10.1203/00006450-199306000-00004

[13] Brogan, P.A., Shah, V., Clarke, L.A., Dillon, M.J. and Klein, N. (2008) T cell activation profiles in Kawasaki syndrome. Clinical \& Experimental Immunology, 151, 267-274. doi:10.1111/j.1365-2249.2007.03567.x

[14] Igarashi, H., Hatake, K., Tomizuka, H., Yamada, M., Gunji, Y. and Momoi, M.Y. (1999) High serum levels of M-CSF and G-CSF in Kawasaki disease. British Journal of Haematology, 105, 613-615. doi:10.1046/j.1365-2141.1999.01381.X

[15] Terai, M., Yasukawa, K., Narumoto, S., Tateno, S., Oana, S. and Kohno, Y. (1999) Vascular endothelial growth factor in acute Kawasaki disease. American Journal of Cardiology, 83, 337-339. doi:10.1016/S0002-9149(98)00864-9

[16] Coussens, L.M. and Werb, Z. (2002) Inflammation and cancer. Nature, 420, 860-867. doi:10.1038/nature01322

[17] Mizukane, R., Kadota, J., Yamaguchi, T., Kiya, T., Fukushima, H., Nakatomi, M. and Kohno, S. (2002) An elderly patients with hemophagocytic syndrome due to severe Mycoplasma pneumonia with marked hypercytokinemia. Respiration, 69, 87-91. doi:10.1159/000049377 